\title{
Mate pair sequencing outperforms fluorescence in situ hybridization in the genomic characterization of multiple myeloma
}

\author{
James Smadbeck', Jess F. Peterson², Kathryn E. Pearce², Beth A. Pitel $\mathbb{0}^{2}$, Andrea Lebron Figueroa ${ }^{2}$, Michael Timm³, \\ Dragan Jevremovic ${ }^{3}$, Min Shi ${ }^{3}$, A. Keith Stewart ${ }^{4}$, Esteban Braggio ${ }^{4}$, Daniel L. Riggs ${ }^{4}$, P. Leif Bergsagel $\mathbb{C}^{4}$, \\ George Vasmatzis', Hutton M. Kearney ${ }^{2}$, Nicole L. Hoppman², Rhett P. Ketterling ${ }^{2}$, Shaji Kumar ${ }^{5}$, \\ S. Vincent Rajkumar $\mathbb{0}^{5}$, Patricia T. Greipp ${ }^{2}$ and Linda B. Baughn ${ }^{2}$
}

\begin{abstract}
Fluorescence in situ hybridization (FISH) is currently the gold-standard assay to detect recurrent genomic abnormalities of prognostic significance in multiple myeloma (MM). Since most translocations in MM involve a position effect with heterogeneous breakpoints, we hypothesize that FISH has the potential to miss translocations involving these regions. We evaluated 70 bone marrow samples from patients with plasma cell dyscrasia by FISH and whole-genome mate-pair sequencing (MPseq). Thirty cases (42.9\%) displayed at least one instance of discordance between FISH and MPseq for each primary and secondary abnormality evaluated. Nine cases had abnormalities detected by FISH that went undetected by MPseq including 6 tetraploid clones and three cases with missed copy number abnormalities. In contrast, 19 cases had abnormalities detected by MPseq that went undetected by FISH. Seventeen were MYC rearrangements and two were 17p deletions. MPseq identified 36 MYC abnormalities and 17 (50.0\% of MYC abnormal group with FISH results) displayed a false negative FISH result. MPseq identified 10 cases (14.3\%) with IgL rearrangements, a recent marker of poor outcome, and 10\% with abnormalities in genes associated with lenalidomide response or resistance. In summary, MPseq was superior in the characterization of rearrangement complexity and identification of secondary abnormalities demonstrating increased clinical value compared to FISH.
\end{abstract}

\section{Introduction}

Multiple myeloma $(\mathrm{MM})$ is a plasma cell neoplasm $(\mathrm{PCN})$ representing the second most common hematopoietic malignancy and accounts for $\sim 20 \%$ of all hematologic cancer related deaths in the United States ${ }^{1}$. During the last decade there have been remarkable improvements in the treatment of patients with MM that have resulted in increased survival, including immunomodulatory compounds, proteasome inhibitors, and immunotherapeutic

\footnotetext{
Correspondence: Linda B. Baughn (baughn.linda@mayo.edu)

${ }^{1}$ Center for Individualized Medicine-Biomarker Discovery, Mayo Clinic,

Rochester, MN, USA

${ }^{2}$ Division of Laboratory Genetics, Department of Laboratory Medicine and

Pathology, Mayo Clinic, Rochester, MN, USA

Full list of author information is available at the end of the article.

These authors contributed equally: James Smadbeck, Jess F. Peterson
}

approaches such as monoclonal antibodies ${ }^{2}$. Paralleling the advances in novel therapeutic strategies, characterization of the genomic complexities of MM have significantly improved with the implementation of nextgeneration sequencing (NGS), thus enabling the iden tification of novel single nucleotide variants (SNV), structural rearrangements and copy number abnormalities $(\mathrm{CNA})^{3-11}$. Comprehensive genomic characterization studies such as the Multiple Myeloma Research Foundation (MMRF) CoMMpass Trial and other research studies are necessary for the discovery of novel variants of clinical significance that may lead to improved treatment approaches and prognostication strategies ${ }^{12,13}$.

In contrast to the use of genome-wide NGS strategies employed in the research/investigational trial setting, 
most clinical genomics laboratories rely upon traditional cytogenetic methodologies such as conventional chromosome studies and fluorescence in situ hybridization (FISH) to characterize recurrent cytogenetic abnormalities of prognostic significance. High-risk cytogenetic abnormalities as defined by the Mayo Clinic mSMART 3.0 algorithm ${ }^{14}$ include $t(4 ; 14), t(14 ; 16), t(14 ; 20)$ translocations, $17 \mathrm{p}$ deletions and 1q gains, while standard-risk cytogenetic abnormalities include hyperdiploidy (gains of odd-numbered chromosomes), $t(11 ; 14)$ and $t(6 ; 14)$ translocations ${ }^{15,16}$. A limited number of laboratories evaluate for $M Y C$ and $\mathrm{t}(6 ; 14)$ rearrangements, and detection of $I G K$ and $I g L$ rearrangements is not routinely performed in the clinical setting ${ }^{15}$. Although FISH assays have high sensitivity, are relatively inexpensive compared to NGS techniques and provide input for risk stratification $^{17}$, several limitations exist. They allow for the interrogation of only the regions for which FISH probes are available and multiple FISH probes are needed in order to be comprehensive, with each probe requiring a resourceconsuming validation. More importantly, FISH has the potential to miss cryptic abnormalities, including rearrangements that result in a position effect due to juxtaposition of enhancers near oncogenes ${ }^{18-22}$. Since many translocations identified in MM involve a position effect (i.e., IGH and $M Y C$ ) with heterogeneous breakpoints $^{10,23,24}$ and some CNAs may be cryptic, we hypothesize that some clinical FISH probes used in the characterization of PCNs have a high rate of false negative FISH results.

To test this hypothesis, we evaluated the performance of a genome wide mate-pair sequencing (MPseq) assay in comparison to FISH panel testing for MM. Since MPseq utilizes long input DNA $(2-5 \mathrm{~Kb})$ followed by circularization and fragmentation to the size of paired-end fragments (200-500 bp) that are sequenced at reduced depth, this assay is designed to detect structural rearrangements and CNAs throughout the genome resulting in a costeffective strategy amenable to a clinical genomics laboratory. Furthermore, as MPseq has higher resolution than FISH and is not limited to specific genomic footprints for interrogation, this assay could provide an alternative technique to comprehensively detect structural rearrangements and CNAs in a single assay. Herein we describe the performance, along with the added clinical utility, of MPseq in 70 samples previously characterized by FISH to detect chromosome rearrangements and CNAs in patients with a PCN.

\section{Methods}

\section{Patient samples}

All samples were referred to the Mayo Clinic Genomics Laboratory as part of routine clinical testing and further evaluated by MPseq as part of a Mayo Clinic Institutional
Review Board approved study. There were multiple sources of samples obtained either from fresh or frozen whole bone marrow (BM), or from fixed cell pellets (FCP) from an abnormal BM chromosome study. Some specimens had undergone plasma cell enrichment from fresh whole BM that was either flow sorted or subjected to CD138+ magnetic-enrichment from patients that had an abnormal plasma cell FISH result. Additional methodology, including conventional chromosome analysis and flow cytometry are included in supplemental data.

\section{Fluorescence in situ hybridization}

Plasma cell proliferative disorder FISH (PCPDF) of immunoglobulin (cIg)-stained positive PCs studies were performed as previously described ${ }^{25}$ using the following probes to detect primary and secondary MM abnormalities: monosomy 13 or 13q deletion (Abbott Molecular, Abbott Park, IL), monosomy 17 or TP53 deletion (Abbott Molecular), trisomy 3, 7, 9 or 15 (Abbott Molecular), 1q gain (in house, custom developed), MYC rearrangement (Abbott Molecular), IGH rearrangement (in house, custom developed), $\mathrm{t}(11 ; 14)$ CCND1/IGH (Abbott Molecular), t(4;14)(p16.3;q32) FGFR3/IGH (Abbott Molecular), $\mathrm{t}(6 ; 14)(\mathrm{p} 21 ; \mathrm{q} 32) C C N D 3 / I G H$ (Abbott Molecular), $\mathrm{t}(14 ; 16)$ (q32;q23) IGH/MAF (Abbott Molecular), and t(14;20)(q32; q12) IGH/MAFB (Abbott Molecular). The PCN FISH panel is indicated in supplemental Table 1 with footprints and probe source shown in supplemental Table 2.

\section{Plasma cell enrichment}

BM cells $\left(20 \times 10^{6}\right)$ were lysed in ACK lysis buffer for $5 \mathrm{~min}$. This was followed by 2 wash steps in PBS (lysewash procedure) and the cell pellet was re-suspended in $3 \%$ BSA/PBS. $10 \times 10^{6}$ cells were then incubated for $15 \mathrm{~min}$ with the following antibodies: CD19-PerCP 5.5 (clone SJ25C1, BD Biosciences), CD38-APC (clone REA671, Miltenyi Biotec), CD45-BB515 (clone HI30, BD Biosciences), CD56-PE-Cy7 (clone NCAM16.2, BD Biosciences), CD138-BV421 (clone MI15, BD Biosciences), and CD319-PE (clone REA150, Miltenyi Biotec). The specimen was centrifuged and re-suspended in $1.5 \mathrm{~mL}$ of PBS. Sorting was performed on BD FACSMelody cell sorter (BD Biosciences, San Jose, CA). Sorting streams were defined for each case separately, using gates to include CD138-positive, CD319-positive, CD38-bright, CD56-positive and/or CD45-negative plasma cells, and separate them from normal plasma cells. A minimum of $2 \times 10^{5}$ cells were collected, with the purity of at least $95 \%$, verified by Kaluza software (Beckman Coulter Life Sciences, Indianapolis, IN). In some cases, plasma cells were separated by positive selection using CD138-coated magnetic beads (MACS; Miltenyi Biotec, CA) in a RoboSep system (STEMCELL Technology, Canada) as described in Jang et al. ${ }^{26}$. 


\section{DNA extraction and library preparation}

DNA extraction and mate pair library preparation methods have been previously described ${ }^{18,27,28}$. Briefly, DNA was isolated using either the Qiagen Puregene extraction kit (for samples $<2 \mathrm{~mL}$ ), Autopure LS Automated high quality DNA extraction (for samples $>2 \mathrm{~mL}$ ) or the QIAmp Tissue kit for fixed cell pellet samples. DNA was processed using the Illumina Nextera Mate Pair library preparation kit and sequenced on the Illumina HiSeq 2500 in rapid run mode as described in Aypar, et al. ${ }^{18}$. Pooled libraries were hybridized onto a flow cell (2 samples per lane) and sequenced using 101-basepair reads and paired end sequencing.

Structural variant bioinformatics pipeline and visualization

The sequencing data was analyzed for the detection of structural variants (SVs), which are large genomic changes $(>30 \mathrm{~Kb})$ that involve breakpoint junctions and/or CNAs. The sequencing data was mapped to the reference genome (GRCh38) using BIMA $^{29}$ and the output was analyzed using SVAtools. This set of algorithms can detect and report the breakpoint locations of both junctions and CNAs at high resolution and accuracy (Schematic in supplemental Fig. 1) ${ }^{18,27,28}$. Junctions and CNVs were graphically illustrated using genome, junction and region plots as previously described ${ }^{18,27,30}$.

\section{Results}

\section{Patient characteristics}

A total of 70 cases referred to the Mayo Clinic Genomics Laboratory for routine clinical PCN FISH testing were selected for further evaluation by MPseq (Tables 1,2 and supplemental Table 3). Criteria for inclusion included the type of primary cytogenetic abnormality to ensure representation of each recurring rearrangement and sample source to evaluate various methods of sample attainment, including PC enrichment (Tables 1, 2). The median age was 66 years (range 42-88) demonstrating male predominance with a 1.3:1 (M:F) ratio. Fifty-seven cases $(81.4 \%)$ had either a diagnosis of MM $(N=35)$ or a reason for referral (RFR) of MM or PCN indicated at the time of clinical testing $(N=22)$ (Tables 1,2$)$. Of thirtyfive cases with complete clinical data, 13 (37.1\%) were newly diagnosed (ND) and 22 (62.9\%) had relapsed and/or refractory disease (RR).

\section{MM abnormalities identified by FISH}

Recurrent primary MM cytogenetic abnormalities identified by FISH in samples $1-65$ were $t(11 ; 14)(21.4 \%)$, $\mathrm{t}(4 ; 14)$ (11.4\%), $\mathrm{t}(14 ; 16)(5.7 \%), \mathrm{t}(14 ; 20)(5.7 \%), \mathrm{t}(6 ; 14)$ (2.9\%), and hyperdiploidy (45.7\%) either without an IGH rearrangement $(32.9 \%)$ or with an IGH rearrangement that did not involve CCND1, FGFR3, MAF, MAFB or CCND3 (12.9\%) (Tables 1, 2). Five samples (cases 66-70) had undefined primary abnormalities including one case of tetrapoloidy with a relative 1q gain, one case with monosomies 13 and 14, two cases with monosomy 15 by FISH and a single case with normal FISH results in a patient with a diagnosis of amyloidosis (Tables 1,2). Conventional chromosome studies were performed on 42 (60.0\%) cases and an abnormal PC clone was identified in $33.3 \%$ of the cases with chromosome studies performed (Supplemental Table 3).

We have previously determined tumor content requirements for MPseq requiring 10\% tumor for the detection of structural rearrangements and $25 \%$ tumor for the detection of $\mathrm{CNAs}^{18}$. Since variable and sometimes low clonal PC percentages can be identified in the $\mathrm{BM}$ aspirates of patients with $\mathrm{NDMM}^{31}$, we performed two enrichment strategies for samples with low PCs including magnetic enrichment or flow sorting. For some samples, no enrichment was performed. Thirty-nine (55.7\%) samples with a median 23.0\% PCs were subjected to either flow sorting ( $N$ $=24)$ or $\mathrm{CD} 138+$ magnetic bead for PC enrichment $(N=$ 15). For the remaining 31 samples (44.3\%) with a median $58 \%$ PC, no PC enrichment was performed.

\section{Identification of recurrent, primary cytogenetic abnormalities using MPseq}

To determine the accuracy of MPseq in comparison to our PCN FISH panel (Supplemental Table 1) in the detection of recurrent, primary MM abnormalities (IGH rearrangement and/or hyperdiploidy), we analyzed DNA extracted from either a fixed cell pellet (FCP) from a chromosome study $(n=8)$, from fresh $(n=18)$ or frozen $(n=5) \mathrm{BM}$ aspirates or from fresh BM specimens that had been flow sorted $(n=24)$ or subjected to CD138+ magnetic enrichment $(n=15)$ (Supplemental Fig. 1, Tables 1, 2). For samples 1-65, MPseq confirmed the primary abnormality identified by FISH in each case demonstrating $100 \%$ concordance between both assays for the classification of recurrent, primary cytogenetic abnormalities (Fig. 1). For those cases without evidence of a recurrent, primary abnormality (samples 66-69), MPseq did not identify tetraploidy in case 66 and monosomy 15 in cases 68-69, but identified monosomies 13 and 14 in case 67 and confirmed no recurrent abnormality in case 70 with normal FISH results. As a negative control, no recurrent primary MM abnormalities (MM specific IGH rearrangements and/or hyperdiploidy with gains of odd numbered chromosomes) were identified by MPseq in a previously described cohort of 88 patients with a reason for referral of acute myeloid leukemia (data not shown $)^{18}$.

\section{Comparison of MPseq to FISH for detection of recurrent, secondary abnormalities}

For each primary and secondary abnormality that was identified by either MPseq or FISH, 40 cases $(57.1 \%)$ 


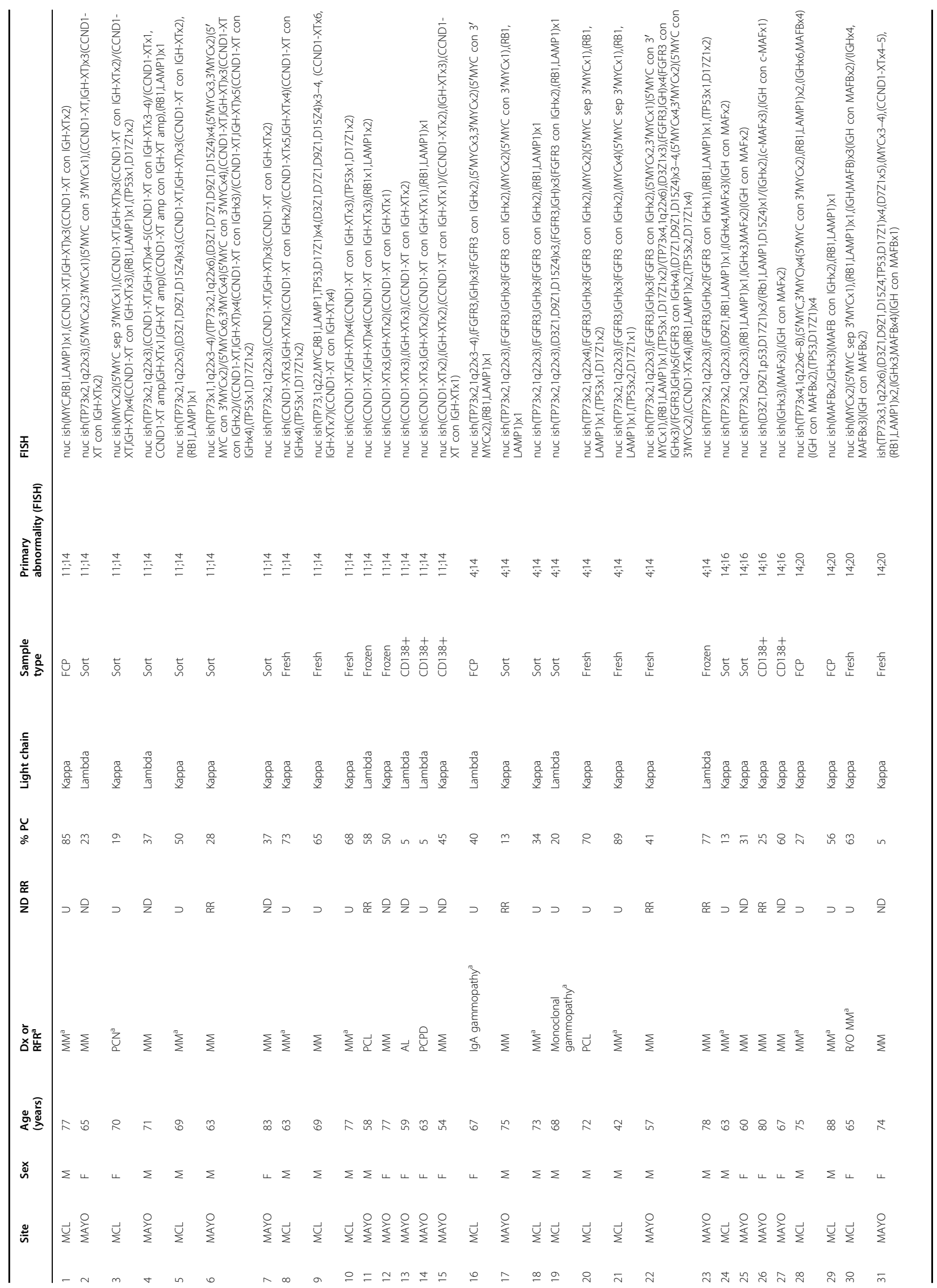




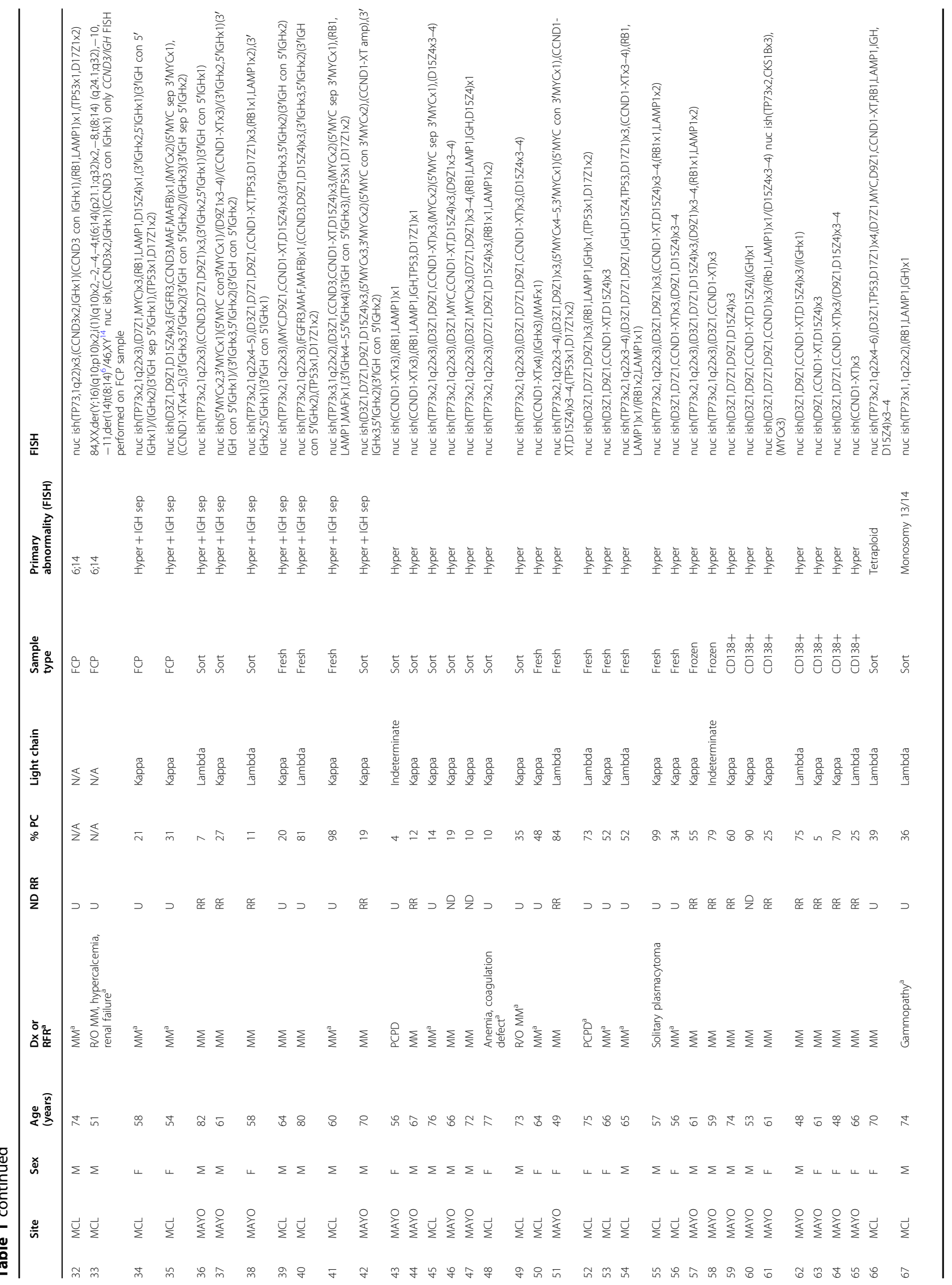




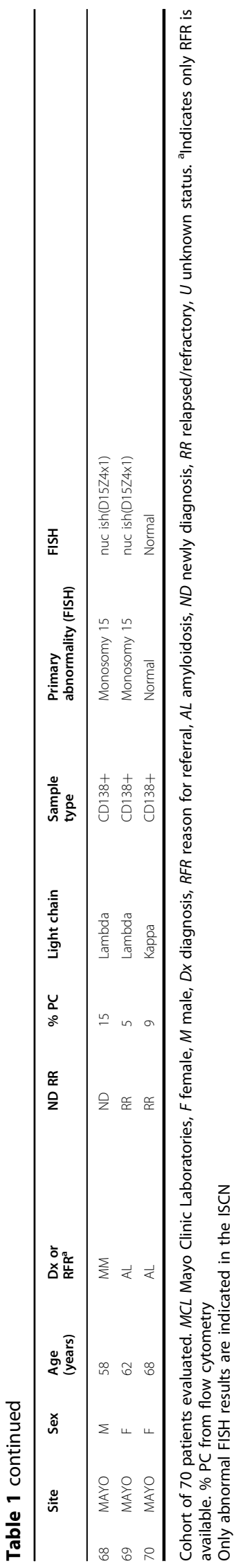

displayed concordance between FISH and MPseq. Thirty cases (42.9\%) displayed at least one instance of discordance between FISH and MPseq (Figs. 1, 2a). Nine of these 30 discordant cases had abnormalities detected by FISH that went undetected by MPseq (Figs. 1, 2a, "FISH advantage"). Of these nine cases, six had a tetraploid clone that was not detectable by MPseq and in three cases MPseq failed to detect CNAs that were identified by FISH (trisomy 3, trisomy 9 and 1q gain). In six cases, FISH identified a CNA involving chromosome 15 that was not confirmed by MPseq. These abnormalities included monosomy 15 identified by FISH without evidence of monosomy 15 by MPseq (cases 26, 47, 68, 69), or one (case 34) or two (case 52) copies of chromosomes 15 identified by FISH in cases with trisomy 15 identified by MPseq (Figs. 1, 2a). In contrast, 19 of the 30 discordant cases had abnormalities detected by MPseq that went undetected by FISH (Figs. 1, 2a, "MPseq advantage"). Of these 19 cases, 17 were $M Y C$ rearrangements and two were $17 \mathrm{p}$ deletions (cases 4 and 21 ), including a $17 \mathrm{p}$ translocation involving the TP53 gene in one case (Figs. 1, 2a).

Increased detection rate of $M Y C$ rearrangements by MPseq

From 70 total cases, we identified 36 cases (51.4\%) that displayed a $M Y C$ rearrangement by MPseq (Fig. 1). Of these 36 cases, 34 had FISH data evaluating the MYC locus. Seventeen cases (50.0\% of $M Y C$ abnormal group with FISH results) displayed a false negative $M Y C$ FISH result where a MYC rearrangement was identified by MPseq, but was negative by FISH (Fig. 1). The most common partner gene/ enhancer segment identified were IGH $(n=7)$, FAM46C ( $=5)$, IGK $(n=4), N S M C E 2(n=4), T X N D C 5(n=4)$ and IGL $(n=4)$ (Table 3$)$. Of the $36 M Y C$ rearrangements, multiple mechanisms resulting in positioning of $M Y C$ near enhancer sequences including small insertions, inversions, simple, balanced or complex translocations were identified (Table 3). The most common method of rearrangement identified in 15 cases included a small insertion of enhancer sequences near the $M Y C$ gene or, alternately, the insertion of $M Y C$ near enhancer sequences. These insertions typically involve the duplication of genetic material of similar size at both the source location and the insertion location, whereby the source DNA is inserted between flanking duplications at the insertion location ${ }^{32}$ (Fig. 2b). Thirteen of these 15 insertion cases co-occurred with hyperdiploidy (hyperdiploidy only or hyperdiploidy with IGH separation) and two of these cases were identified by FISH studies. Of the 17 $M Y C$ cases that were missed by FISH, 11 represented these small insertions (Table 3).

\section{Detection of additional genomic alterations by MPseq that are not evaluated by FISH}

We next evaluated for the presence of rearrangements involving non-recurrent IGH MM partners (excluding 
Table 2 Patient characteristics

\begin{tabular}{|c|c|}
\hline \multicolumn{2}{|l|}{ Total $(N=70)$} \\
\hline Characteristic & $N(\%)$ \\
\hline \multicolumn{2}{|l|}{ Sex } \\
\hline Male & $40(57.1)$ \\
\hline Female & $30(42.9)$ \\
\hline \multicolumn{2}{|l|}{ Age } \\
\hline Median & 66 years \\
\hline Range & $42-88$ years \\
\hline $40-49$ & $4(5.7)$ \\
\hline $50-59$ & $14(20.0)$ \\
\hline $60-69$ & $26(37.1)$ \\
\hline 70-79 & $21(30.0)$ \\
\hline $80-89$ & $5(7.1)$ \\
\hline \multicolumn{2}{|l|}{ Diagnosis or RFR } \\
\hline MM, PCN diagnosis or RFR & $57(81.4)$ \\
\hline Amyloidosis & $3(4.3)$ \\
\hline Plasma cell leukemia & $2(2.9)$ \\
\hline Plasma cell proliferative disorder & $3(4.3)$ \\
\hline Other & $5(7.1)$ \\
\hline \multicolumn{2}{|l|}{ Site } \\
\hline Mayo Clinic-local & $37(52.9)$ \\
\hline Mayo Clinic Laboratories-outside & $33(47.1)$ \\
\hline \multicolumn{2}{|l|}{$P C$ percentage $(N=68)$} \\
\hline Median & $35.5 \%$ \\
\hline Range & $4-99 \%$ \\
\hline $4-19$ & $19(27.9)$ \\
\hline $20-39$ & $19(27.9)$ \\
\hline $40-59$ & $11(16.2)$ \\
\hline $60-79$ & $12(17.6)$ \\
\hline $80-99$ & $7(10.3)$ \\
\hline \multicolumn{2}{|l|}{ Sample type } \\
\hline No enrichment & $31(44.3)$ \\
\hline Fixed cell pellet (FCP) & 8 (11.4) median PCs (35.5) \\
\hline Fresh sample & 18 (25.7) median PCs (66.5) \\
\hline Frozen sample & 5 (7.1) median PCs (58.0) \\
\hline Enrichment & $39(55.7)$ \\
\hline Flow sorting & 24 (34.3) median PCs (19.5) \\
\hline CD138+ magnetic & 15 (21.4) median PCs (25.0) \\
\hline \multicolumn{2}{|l|}{ Light chain } \\
\hline Kappa & $46(65.7)$ \\
\hline Lambda & $20(28.6)$ \\
\hline Indeterminate or unknown & $4(5.7)$ \\
\hline \multicolumn{2}{|l|}{ Primary cytogenetic abnormality } \\
\hline$t(11 ; 14)$ & $15(21.4)$ \\
\hline $\mathrm{t}(4 ; 14)$ & $8(11.4)$ \\
\hline$t(14 ; 16)$ & $4(5.7)$ \\
\hline$t(14 ; 20)$ & $4(5.7)$ \\
\hline$t(6 ; 14)$ & $2(2.9)$ \\
\hline Hyperdiploid only & $23(32.9)$ \\
\hline $\begin{array}{l}\text { Hyperdiploid with an unknown IGH } \\
\text { rearrangement }\end{array}$ & $9(12.9)$ \\
\hline Tetraploid without primary abnormality & $1(1.4)$ \\
\hline Monosomy 13/14 alone & $1(1.4)$ \\
\hline Monosomy 15 alone & $2(2.9)$ \\
\hline Normal & $1(1.4)$ \\
\hline
\end{tabular}

Table 2 continued

\begin{tabular}{ll}
\hline Total $(\boldsymbol{N}=\mathbf{7 0})$ & \\
\hline Characteristic & $\boldsymbol{N}(\%)$ \\
\hline Conventional chromosome study & \\
Not performed & $28(40.0)$ \\
Performed & $42(60.0)$ \\
Normal or loss of $Y$ & $27(38.6$ of total, 64.3 of \\
performed) & $14(20.0$ of total, 33.3 of \\
Abnormal with PC abnormalities & performed) \\
Abnormal with non-PC abnormalities & $1(1.4$ of total, 2.4 of performed)
\end{tabular}

Patient characteristics of the 70 patients within the cohort evaluated

CCND1, FGFR3, MAF, MAFB or CCND3) and the IGK and IGL loci by MPSeq. There were 19 additional IGH rearrangements identified in 18 cases (25.7\% of cohort) with partner chromosomes at $8 \mathrm{q} 24.21(M Y C)(n=7)$ as the only recurrent rearrangement (Table 4, Fig. 3). Of the nine cases classified as "hyperdiploidy with IGH separation", an IGH partner was identified in six cases, while the other three cases had a loss within the IGH locus. Two cases (cases 50 and 54) classified as hyperdiploidy without an IGH rearrangement to one of the common partner chromosomes had the "small insertion" type of MYC/IGH rearrangement (Tables 3, 4, Fig. 3). There were three cases with a CCND1 rearrangement to a locus other than IGH (IGK/CCND1 in case 43 , IGL/CCND1 in case 4 and BRINP3/CCND1 in case 57) that had additional copies of CCND1 observed by FISH in case 4 and 43 . FISH for CCND1 was not performed in case 57 and in case 4, the signal pattern for CCND1 was scored as amplification (Table 4, Figs. 1, 3).

There were five cases with IGK rearrangements $(7.1 \%$ of cohort) mainly with partner chromosome 8q24.21 (MYC) $(\mathrm{n}=4)$ and a single case with partner chromosome at 11q13.3 (CCND1) (Table 4, Fig. 3). In addition, 10 cases (14.3\% of cohort) had IGL rearrangements with partner chromosomes at $8 \mathrm{q} 24.21 \quad(M Y C) \quad(\mathrm{n}=4), \quad 11 \mathrm{q} 13.3$ $(C C N D 1) \quad(\mathrm{n}=1), \quad 8 \mathrm{q} 24.22 \quad(\mathrm{n}=3) \quad$ (putative target ST3GAL1/NDRG1), 3q26.2 (MECOM) $\quad(\mathrm{n}=1)$ and 17q25.1 (GRB2) ( $\mathrm{n}=1)$ (Table 4, Fig. 3). Of these 15 cases with either an IGK or IGL rearrangement, 12 (80.0\%) cooccurred with hyperdiploidy (hyperdiploidy only or hyperdiploidy with IGH separation).

We explored alterations in additional genes contributing to dysregulation of multiple pathways such as WNT or NF-kB signaling including genes $C Y L D$ at 16q12.1, $B I R C 2$ and BIRC3 at $11 \mathrm{q} 22,2, N F K B 1$ at $4 \mathrm{q} 24, N F K B 2$ at 10q24.32, TRAF2 at 9q34.3, TRAF3 at $14 q 32.32$ and $M A P 3 K 14 / N I K$ at $17 \mathrm{q} 21.31$ or other tumor suppressor genes such as CDKN2C (p18) at $1 \mathrm{p} 32.3$ or TENT5C/ FAM46C at $1 \mathrm{p} 12^{33,34}$ (Table 5, Fig. 3). Twenty-five cases 


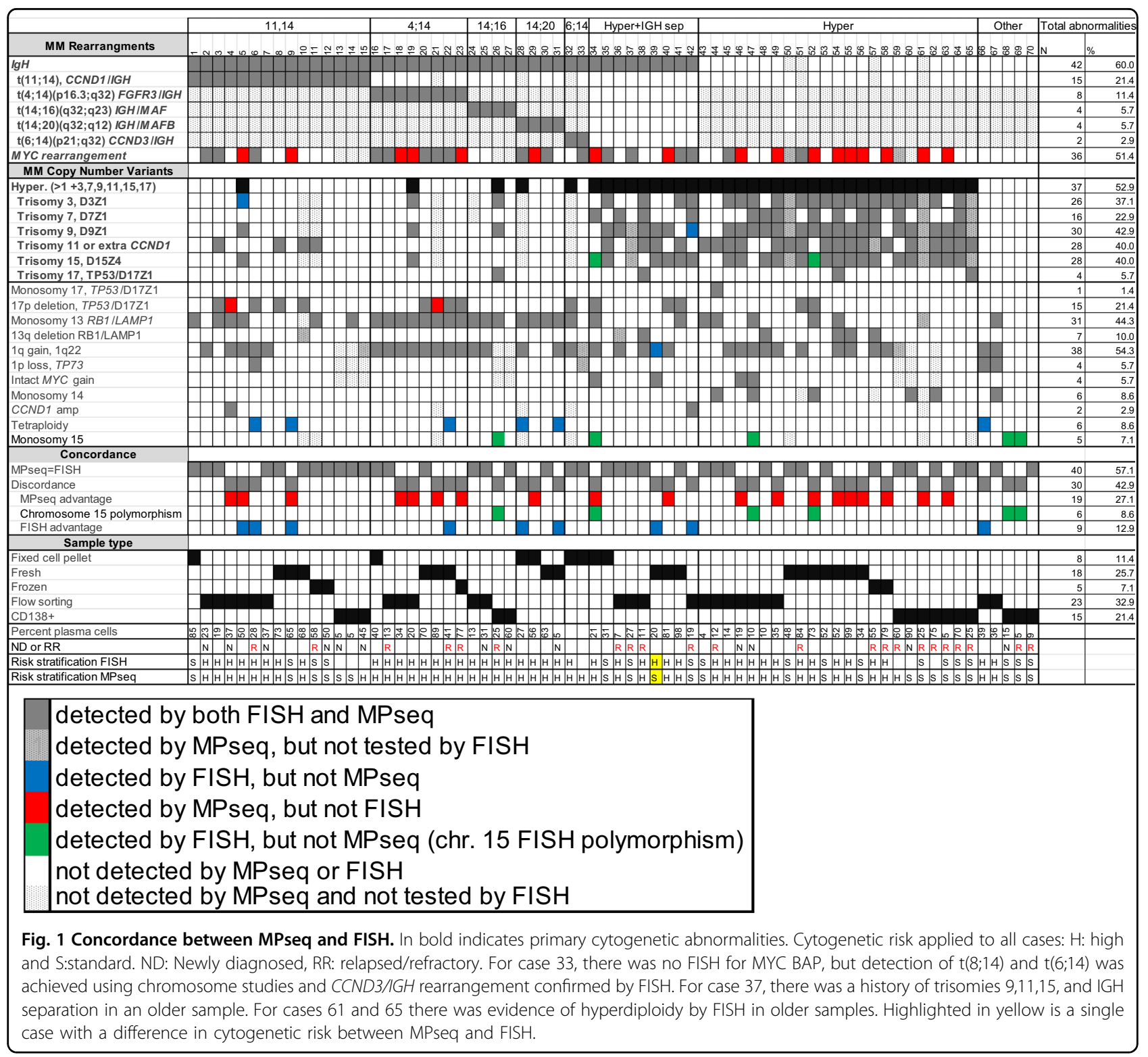

(35.7\%) had an alteration in TENT5C/FAM46C with 6 cases with translocations (five of these to $M Y C$ ) and 19 cases had a heterozygous deletion of TENT5C/FAM46C ranging in size from $3.4 \mathrm{Mb}$ to $120 \mathrm{Mb}$. Nineteen cases (27.1\%) had alterations in CDKN2C and/or FAF1. Fourteen were heterozygous deletions involving $C D K N 2 C$ ranging in size from $587 \mathrm{~Kb}$ to $120 \mathrm{Mb}$, four cases had focal biallelic CDKN2C and FAF1 deletions (Supplemental Fig. 2A, case \#5) and one case had a heterozygous $655 \mathrm{~Kb} F A F 1$ deletion without a CDKN2C deletion (Table 5, Fig. 3). Ten cases had deletions of TRAF3 with 5 as heterozygous deletions and five as biallelic deletions (Supplemental Fig. 2B, case \#62) and a single case had a $92.9 \mathrm{~Kb}$ heterozygous deletion of TRAF2 (Table 5, Fig. 3). Twenty-eight cases had deletions of CYLD $(40 \%$ of cohort) with 24 cases having heterozygous deletions ranging in size from $634 \mathrm{~Kb}$ to $90.3 \mathrm{Mb}$ with the majority representing large $16 \mathrm{q}$ deletions and four cases will smaller biallelic deletions (Table 5, Fig. 3, Supplemental Fig. 2C, case \#40). Additional alterations in MAP3K14 were observed in four cases (three as heterozygous deletions and one as a $735 \mathrm{~Kb}$ gain), heterozygous deletion of $N F K B 1$ in seven cases, heterozygous deletion of NFKB2 in 6 cases and a heterozygous and homozygous $B I R C 2$ and BIRC3 deletions in two separate cases (Table 5, Fig. 3).

Evaluation for loss of function alterations of genes that have been associated with lenalidomide response or resistance (CRBN, IKZF1 and IKZF3) identified $10.0 \%$ of the cohort had either a CRBN, IKZF1 and IKZF3 gene alteration (Table 5, Fig. 3, Supplemental Fig. 2D, case 


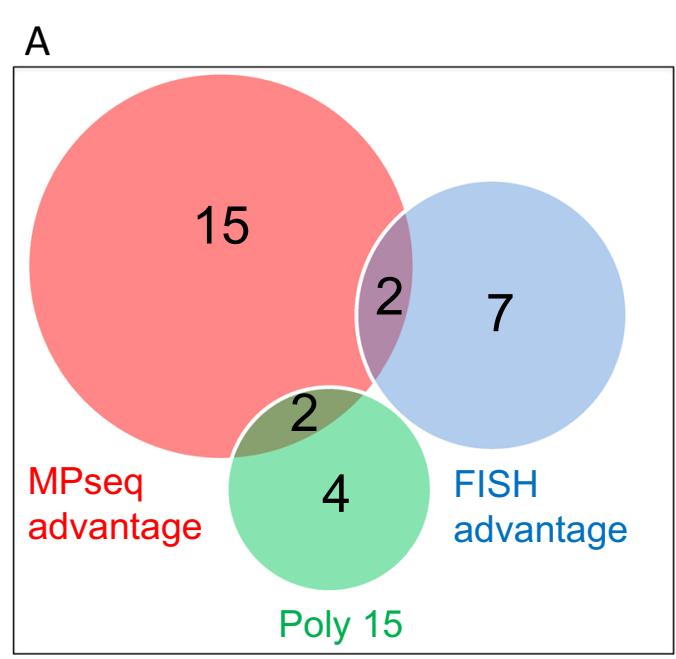

\begin{tabular}{|lc|c|}
\hline Discordance Summary & \multicolumn{2}{c|}{ Total } \\
\hline FISH advantage (detected by FISH, but not MPseq) only & 7 \\
MPseq missed tetraploidy & 5 & \\
MPseq missed 1q gain & 1 & \\
MPseq missed trisomy 9 & 1 & \\
\hline FISH and MPseq advantage & 1 & \\
FISH missed MYC, MPseq missed trisomy 3 & 1 & \\
FISH missed MYC, MPseq missed tetraploidy & 1 & \\
\hline MPseq advantage (detected by MPseq, but not FISH) only & 15 \\
FISH missed MYC & 13 & \\
FISH missed TP53 & 2 & \\
\hline MPseq advantage and poly 15 & 2 \\
FISH missed MYC & 2 & \\
\hline Polymorphism 15 only & 4 \\
\hline \multicolumn{2}{|c|}{30} \\
\hline
\end{tabular}

B

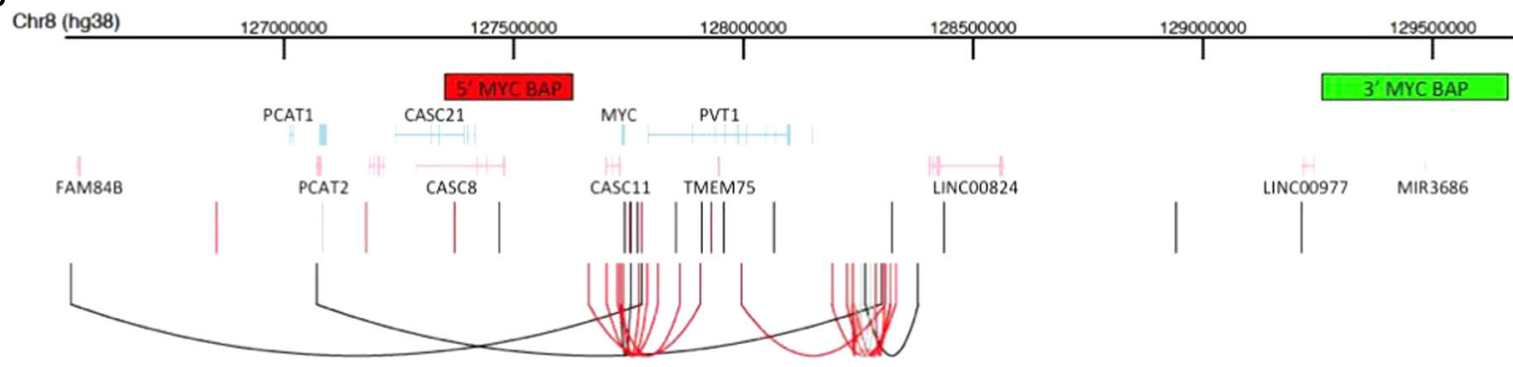

Fig. 2 Discordance summary and MYC breakpoint locations. a Total number of cases with evidence of MPseq advantage, FISH advantage and polymorphism of chromosome 15. b The location of breakpoints in the MYC locus across all cases are depicted as vertical lines (black if the MYC alteration was detected by FISH, light gray if it was not tested by FISH, and red if it was undetected by FISH). In cases where multiple breakpoints were found in the MYC locus, the lines are connected by an arc. The locations of the MYC BAP probes used for FISH detection are shown at the top ( $5^{\prime}$ in red, $3^{\prime}$ in green) and gene locations are shown in the middle (forward strand in light blue, reverse strand in pink).

\#58). Specifically, five cases had a heterozygous deletion of $C R B N$ ranging in size from $457 \mathrm{~Kb}$ to $130.5 \mathrm{Mb}$ including case \#58 that had both a 7.1 Mb deletion encompassing $C R B N$ and a $223 \mathrm{~Kb} I K Z F 1$ duplication with insertion of $I K Z F 1$ into 10q25.2. Two cases had a heterozygous deletion that included IKZF3 (9.1 Mb and $45.7 \mathrm{Mb})$.

\section{Discussion}

Most clinical laboratories employ FISH analysis of CD138 enriched plasma cells as the preferred methodology in order to identify recurrent primary and secondary genomic abnormalities of prognostic and therapeutic significance in patients with $\mathrm{PCNs}^{15}$. The majority of these laboratories utilize only a limited FISH panel with many focusing on high risk abnormalities defined by the revised International Staging System (R-ISS) including 1q gain, $t(4 ; 14), t(14 ; 16)$ or $17 p$ deletion ${ }^{15}$. Some laboratories have incorporated the use of chromosomal microarray analysis in the detection of CNAs such as hyperdiploidy, $17 \mathrm{p}$ deletions and 1q gains, however microarray studies are unable to identify balanced structural rearrangements necessitating the use of other methodologies in the detection of IGH rearrangements ${ }^{15}$. It has also become increasingly apparent that some FISH probes, such as those targeting $M Y C$ rearrangements, display evidence of false negative results ${ }^{18-22}$. In addition, FISH panels for PCNs are variable between individual laboratories, provide a limited view of the whole genome and may not always reflect genomic complexity. Given that multiple research studies and investigational trials have used NGS based techniques to identify CNAs, SNVs along with structural rearrangements, we sought to explore the feasibility of employing an NGS technique in the detection of CNAs and structural rearrangements as a FISH replacement assay within a clinical genomics laboratory.

We describe the performance and added utility of a whole genome NGS based strategy, MPseq, in comparison to the current gold standard FISH approach in the evaluation of patients with PCNs. While MPseq and FISH displayed equal performance in the ability to classify the presence or absence of a recurrent, primary cytogenetic subtype (i.e. hyperdiploidy or specific IGH 
Table 3 Genetic information of secondary alterations involving MYC.

\begin{tabular}{|c|c|c|c|c|c|c|c|c|c|}
\hline Case & $\mathrm{FISH}$ & Type & Junction & Chr Partner & Pos partner & Pos $M Y C$ & $\begin{array}{c}\text { MYC } \\
\text { Loc }\end{array}$ & Gene Pair & Primary \\
\hline \multirow{2}{*}{2} & & \multirow{2}{*}{ Complex } & \multirow{2}{*}{2} & 11 & 26066986 & 127732671 & \multirow{2}{*}{$\mathrm{E}$} & \multirow{2}{*}{ ANO3 } & \multirow{8}{*}{$t(11 ; 14)$} \\
\hline & & & & 17 & 47142366 & 127755273 & & & \\
\hline 3 & & Balanced & 1 & 2 & 88838370 & 127770919 & $R$ & IGK & \\
\hline 5 & & Complex & 1 & 6 & 7913150 & 127180114 & $\mathrm{~L}$ & TXNDC5 & \\
\hline \multirow{2}{*}{6} & & \multirow{2}{*}{ Balanced } & \multirow{2}{*}{2} & 11 & 25654318 & 126537887 & \multirow{2}{*}{ E } & \multirow{2}{*}{ ANO3 } & \\
\hline & & & & 11 & 26079246 & 127779590 & & & \\
\hline 9 & & Balanced & 1 & 14 & 105862404 & 126854184 & L & $I G H$ & \\
\hline 16 & & Tandem Dup & 1 & 8 & 97622416 & 127755097 & $R$ & $M T D H$ & \\
\hline 17 & & Balanced & 1 & 4 & 63788054 & 128942926 & $\mathrm{R}$ & TECRL & \multirow{10}{*}{$\mathrm{t}(4 ; 14)$} \\
\hline 18 & & Tandem Dup & 1 & 8 & 125268967 & 127780538 & $R$ & NSMCE2 & \\
\hline \multirow{2}{*}{19} & & \multirow{2}{*}{ Small Insertion } & \multirow{2}{*}{2} & 6 & 7929621 & 128320023 & \multirow{2}{*}{$\mathrm{R}$} & \multirow{2}{*}{ TXNDC5 } & \\
\hline & & & & 6 & 7964553 & 128226141 & & & \\
\hline 20 & & Complex & 1 & 14 & 105577910 & 128067445 & $\mathrm{R}$ & $I G H$ & \\
\hline & & & & 8 & 128296726 & 127956701 & $R$ & & \\
\hline 21 & & Complex & 2 & 8 & 115196922 & 128311995 & $\mathrm{~L}$ & CSMD3 & \\
\hline 22 & & Translocation & 1 & 22 & 22898779 & 129213302 & $R$ & IGL & \\
\hline 23 & & Small Incertion & 2 & 8 & 97602388 & 128244496 & $R$ & MTOH & \\
\hline 23 & & small Insertion & 2 & 8 & 97631703 & 128238180 & $R$ & MIDH & \\
\hline 28 & & Translocation & 1 & 2 & 172598535 & 128436028 & $\mathrm{R}$ & $P D K 1$ & \\
\hline 29 & & Inversion & 1 & 8 & 125294077 & 127754738 & $R$ & NSMCE2 & $\mathrm{t}(14 ; 20)$ \\
\hline 30 & & Balanced & 1 & 1 & 117855602 & 127910156 & $R$ & FAM46C & \\
\hline 33 & & Translocation & 1 & 14 & 105850372 & 127085883 & $\mathrm{~L}$ & $I G H$ & $t(6 ; 14)$ \\
\hline 34 & & Small Incertion & 2 & 1 & 117746520 & 128192712 & $R$ & FAM46C & \\
\hline 34 & & Shall insertion & 2 & 20 & 40219115 & 128332443 & $\mathrm{~K}$ & $M A F B$ & \\
\hline 35 & & Small Insertion & 2 & 22 & 23048204 & 128265766 & $R$ & $|G|$ & \\
\hline 35 & & Smail insertion & 2 & 22 & 22879587 & 128381540 & R & IGL & Hyper + \\
\hline 37 & & Complex & 1 & 8 & 125349863 & 128299421 & $\mathrm{R}$ & NSMCE2 & \\
\hline 40 & & Translocation & 1 & 14 & 105620039 & 127372549 & L & $I G H$ & \\
\hline 41 & & Translocation & 1 & 2 & 88796421 & 128324052 & $\mathrm{R}$ & IGK & \\
\hline 42 & & Translocation & 1 & 14 & 105729139 & 127853468 & $\mathrm{~L}$ & $I G H$ & \\
\hline 45 & & Small Insertion & 1 & 22 & 22914490 & 127742293 & $R$ & $I G L$ & \\
\hline & & Small Insertion & 2 & 6 & 7911365 & 128320871 & $R$ & $T X N D C 5$ & \\
\hline 46 & & small Insertion & 2 & 6 & 7994873 & 128243759 & $R$ & IXNNDCS & \\
\hline & & Translocation & 1 & 2 & 88806363 & 127710025 & $R$ & IGK & Hvaner \\
\hline 49 & & SmallIncertion & 2 & 6 & 7983613 & 127815314 & $R$ & TYNIRC5 & пурег \\
\hline 49 & & Smail insertion & 2 & 6 & 7837493 & 127704213 & R & IXNNDCS & \\
\hline 50 & & Small Insertion & 2 & 14 & 105562884 & 128276113 & $R$ & $I G H$ & \\
\hline 50 & & Small Insertion & 2 & 14 & 105611952 & 128243175 & $\mathrm{R}$ & & \\
\hline 51 & & Amplification & 1 & 8 & 125363400 & 127470538 & $\mathrm{~L}$ & NSMCE2 & \\
\hline 52 & & Small Incertion & 2 & 3 & 46288493 & 127790484 & $\mathrm{~F}$ & $C C R 3$ & \\
\hline 52 & & Smál misertiour & 2 & 3 & 46331780 & 127733550 & ᄃ & C & \\
\hline 54 & & Small Insertion & 2 & 14 & 105564852 & 127737562 & $E$ & $I G H$ & \\
\hline & & & & 14 & 105586305 & 127775237 & E & & \\
\hline 55 & & Small Insertion & 2 & 22 & 23019052 & 127664055 & $E$ & $|G|$ & \\
\hline & & & & 22 & 22912000 & 127907000 & & & \\
\hline 56 & & Small Insertion & 2 & 1 & 117615759 & 127995692 & $R$ & FAM46C & \\
\hline 56 & & Smail Insertion & 2 & 1 & 117851083 & 128308588 & $R$ & FAM46C & \\
\hline 58 & & Small Insertion & 2 & 2 & 88671060 & 127726700 & $E$ & IGK & \\
\hline 58 & & Small Insertion & & 2 & 88854911 & 127862698 & & & \\
\hline 59 & & Balanced & 1 & 1 & 117670599 & 127931065 & $\mathrm{R}$ & FAM46C & \\
\hline 61 & & Small Insertion & 2 & 1 & 117653222 & 128307014 & $R$ & FAM46C & \\
\hline 61 & & Small Insertion & 2 & 1 & 117665080 & 128287350 & $R$ & FAM46C & \\
\hline 62 & & Smallinsertion & 2 & 11 & 73143438 & 128285026 & $R$ & $\mathrm{E}$ & \\
\hline 62 & & Smail insertion & 2 & 11 & 73166000 & 128255000 & R & FCHSDL & \\
\hline
\end{tabular}

\begin{tabular}{|l|l|}
\hline Detected by both FISH and MPseq \\
Detected by MPseq, but not tested by FISH \\
Detected by MPseq, but not FISH \\
\hline
\end{tabular}

For each case where a secondary alteration involving MYC was found, the relevant genomic information is provided for the junction(s). The case column is the case number. The FISH column indicates whether or not the MYC FISH test detected the secondary alteration (dark gray-detected by both FISH, light gray-detected by MPseq but not tested by FISH and red-detected by MPseq only). The type column is the type of alteration involved with MYC classified as either a balanced event, a tandem duplication, a translocation, an inversion, part of an amplification, part of a small insertion motif, a complex event, or ND where it was not possible to definitively classify the alteration. The Junction column is the number of junctions involved directly in the alteration, either 1 or 2 . The Chr Partner and Pos Partner columns are the chromosome and position location (GRCh38) of the partner breakpoints that are part of alteration. The Pos MYC and MYC Loc columns give the position of the breakpoint in the MYC locus and whether the alteration is to the left, right, or encompassing ( $\mathrm{L}, \mathrm{R}$, or $\mathrm{E})$ the $M Y C$ gene, respectively. The Gene Pair column is the gene that is found at or near the partner breakpoint location. The Primary column is the primary alteration for the case 
Table 4 IGH, IGK, and IGL partner genes.

\begin{tabular}{|c|c|c|c|}
\hline Case & $\begin{array}{l}\text { IGH partner } \\
\text { chromosome }\end{array}$ & $\begin{array}{l}\text { Putative } \\
\text { gene target }\end{array}$ & $\begin{array}{l}\text { Primary } \\
\text { abnormality }\end{array}$ \\
\hline 5 & $14 q 24.3$ & BATF & $11 ; 14$ \\
\hline 6 & 19p13.2 & TYK2 & $11 ; 14$ \\
\hline 8 & $11 q 14.1$ & RAB39 & $11 ; 14$ \\
\hline 9 & 1 p35.3 & PTPRU & $11 ; 14$ \\
\hline 9 & $8 q 24.21$ & MYC & $11 ; 14$ \\
\hline 10 & $20 q 11.21$ & COMMD7 & $11 ; 14$ \\
\hline 11 & $22 q 13.1$ & $P O L R 2 F$ & $11 ; 14$ \\
\hline 15 & $2 \mathrm{p} 24.3$ & MYCN & $11 ; 14$ \\
\hline 20 & $8 q 24.21$ & MYC & $4 ; 14$ \\
\hline 30 & $5 p 15.33$ & TERT & $14 ; 20$ \\
\hline 33 & $8 q 24.21$ & MYC & $6 ; 14$ \\
\hline 34 & $7 q 32.1$ & Unknown & Hyper + IGH sep \\
\hline 36 & Xq32.33 & MTMR1 & Hyper + IGH sep \\
\hline 37 & $14 q 24.3$ & DPF3 & Hyper + IGH sep \\
\hline 40 & $8 q 24.21$ & MYC & Hyper + IGH sep \\
\hline 41 & $9 p 13.2$ & PAX5 & Hyper + IGH sep \\
\hline 42 & $8 q 24.21$ & MYC & Hyper + IGH sep \\
\hline 50 & $8 q 24.21$ & MYC & Hyper \\
\hline 54 & $8 q 24.21$ & MYC & Hyper \\
\hline Case & $\begin{array}{l}\text { IGK partner } \\
\text { chromosome }\end{array}$ & $\begin{array}{l}\text { Putative } \\
\text { gene target }\end{array}$ & $\begin{array}{l}\text { Primary } \\
\text { abnormality }\end{array}$ \\
\hline 3 & $8 q 24.21$ & MYC & $11 ; 14$ \\
\hline 41 & $8 q 24.21$ & MYC & Hyper + IGH sep \\
\hline 43 & $11 q 13.3$ & CCND1 & Hyper \\
\hline 46 & $8 q 24.21$ & MYC & Hyper \\
\hline 58 & $8 q 24.21$ & MYC & Hyper \\
\hline Case & $\begin{array}{l}\text { IGL partner } \\
\text { chromosome }\end{array}$ & $\begin{array}{l}\text { Putative } \\
\text { gene target }\end{array}$ & $\begin{array}{l}\text { Primary } \\
\text { abnormality }\end{array}$ \\
\hline 4 & $11 \mathrm{q} 13.3$ & CCND1 & $11 ; 14$ \\
\hline 22 & $8 q 24.21$ & MYC & $4 ; 14$ \\
\hline 35 & $8 q 24.21$ & MYC & Hyper + IGH sep \\
\hline 39 & $3 q 26.2$ & MECOM & Hyper + IGH sep \\
\hline 45 & $8 q 24.21$ & MYC & Hyper \\
\hline 50 & $17 q 25.1$ & GRB2 & Hyper \\
\hline 55 & $8 q 24.21$ & MYC & Hyper \\
\hline 56 & $8 q 24.22$ & ST3GAL1/NDRG1 & Hyper \\
\hline 61 & $8 q 24.22$ & ST3GAL1/NDRG1 & Hyper \\
\hline 63 & $8 q 24.22$ & ST3GAL1/NDRG1 & Hyper \\
\hline
\end{tabular}

Partner genes associated with IGH, IGK, and IGL showing cytogenetic location and putative target genes. Hyper: Hyperdiploidy only. Hyper+IGH sep: Hyperdiploidy with IGH separation

rearrangement), MPseq was superior compared to FISH in the characterization of rearrangement complexity, identification of secondary abnormalities, resolution of atypical FISH results and identification of novel abnormalities of prognostic significance not targeted by traditional FISH panels. Many samples chosen for this study had a high plasma cell burden (median 36\% PCs) and $\sim 33 \%$ of cases were obtained from fresh or frozen samples that did not require enrichment.

An advantage to using a whole genome NGS technique like MPseq is the ability to identify rearrangements using an unbiased approach. Other laboratories have developed and validated NGS methodologies utilizing targetenrichment approaches for PCNs allowing a custom target pull down of limited genomic regions ${ }^{13,35-37}$. While these targeted approaches have reduced cost and simplified analysis workflows, a genome wide approach utilizing long-insert whole genome sequencing employed by the MMRF CoMMpass Study in their Seq-FISH analysis has demonstrated improved sensitivity with similar specificity in relation to clinical FISH testing ${ }^{38}$. Although MPseq is similar to Seq-FISH with regard to a whole genome sequencing approach, a significant limitation to the current MPseq strategy is the inability to identify SNVs. This limitation can be resolved with deeper and faster sequencing, coupled with reduced sequencing costs. An integrated genomic analysis incorporating structural variation, CNAs, and SNVs together may lead to enhanced prognostication ${ }^{13}$. Of practical consideration is the $\sim$ twofold increased cost and "turn-around-time" of reporting of clinical grade testing for MPseq compared to a comprehensive FISH panel; although we anticipate over time the cost and time of reporting for NGS approaches will continue to be reduced.

Another limitation to the use of MPseq is the inability to identify rearrangements in highly repetitive regions of the genome containing constitutive heterochromatin such as those involving telomeres, centromeres, and in regions near the centromeres of chromosomes 1,9 , and 16 and in the $\mathrm{Y}$ chromosome ${ }^{27}$. This limitation may be reflected by the inability of MPseq to identify apparent trisomies in 2 cases (cases 5 and 42) with evidence of hyperdiploidy. Case 5 displayed a gain of a structurally abnormal chromosome 3 by conventional chromosome studies. Since the centromere regions that are targeted by the FISH probes are not covered by MPseq, it is unclear whether a small gain or presence of a polymorphism of these regions are present without evidence of a bona fide trisomy or whether the trisomy was present at a subclonal level below the limit of detection by MPseq $(<25 \% \text { for CNAs })^{18}$. Polymorphisms of the acrocentric chromosome 15 have also been reported ${ }^{39}$ and are observed in FISH analysis of PCNs in our laboratory (data not shown). Discrepancies involving chromosome 15 are present in 6 of 70 cases in this study demonstrated by either a monosomy 15 FISH result with normal chromosome $15 \mathrm{~s}$ by MPseq or either a normal or monosomy 15 FISH result with trisomy 15 by 


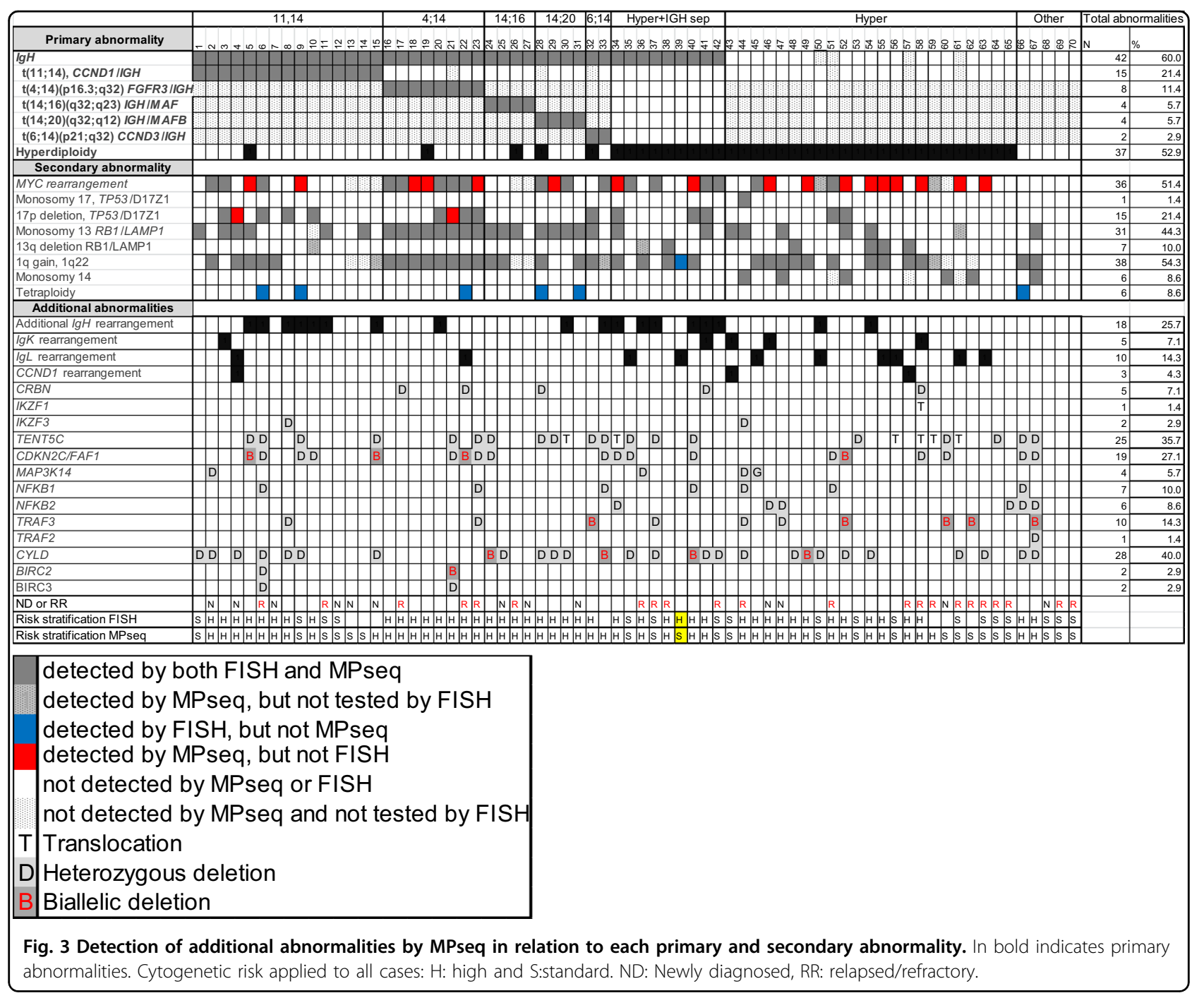

MPseq. Since MPseq does not rely on detection of only the centromere region like FISH, analysis of copy number changes throughout the whole chromosome can be useful to interpret the presence or absence of a trisomy. On the other hand, any missed hyperdiploid cases may be of less relevance as hyperdiploidy can be detected by flow cytometric methods ${ }^{40}$.

MPseq is not currently used in the detection of copyneutral LOH or ploidy. As a result, MPseq did not identify any cases as tetraploid, an abnormality found in approximately $6 \%$ of patients with MM that has been associated with high-risk genomic abnormalities and with poor prognosis in $\mathrm{NDMM}^{41}$. Six patients in our cohort had evidence of tetraploidy and 5 of these cases had tetraploidy in combination with high risk cytogenetics (Fig. 1 ), which were identified by MPseq. For case 39, it is unclear why MPseq failed to identify a 1q gain. No evidence of a duplication involving chromosome 1q (chr. 1:155122503-155571708) was identified by MPseq and based on FISH data, the 1q gain did not appear to be subclonal, however this sample was extracted from unsorted bone marrow with $20 \%$ clonal plasma cells which may have contributed to this missed abnormality. Subclonal CNAs and cases with low tumor load have a risk of being missed by MPseq, a risk that also exists when performing FISH.

Of the 30 discordant cases, 19 cases had abnormalities identified by MPseq that were missed by FISH, the majority involving insertional events near the $M Y C$ gene region. In total, $M Y C$ rearrangements were found in $51.4 \%$ of the cases in our cohort which includes NDMM and RRMM. This is consistent with previous reports identifying $M Y C$ rearrangements in 35\% of NDMM with increased frequency in $\mathrm{RRMM}^{10,42}$. MYC rearrangements have been reported as subclonal events and are associated with disease progression ${ }^{3,11,43}$. Approximately $66 \%$ of $M Y C$ rearrangements have been found in association with non-Ig partners resulting in juxtaposition to enhancer 
Table 5 Abnormalities of additional genes of clinical significance

\begin{tabular}{|c|c|c|c|c|c|}
\hline Case & CYLD & Location & Breakpoints & Size (bp) & Primary \\
\hline 1 & $\mathrm{HD}$ & $16 p 13.3-16 q 24.3$ & 0-90338345 & 90338345 & $11 ; 14$ \\
\hline 2 & $\mathrm{HD}$ & $16 q 11.2-16 q 24.3$ & $46454000-90338000$ & 43884000 & $11 ; 14$ \\
\hline 4 & $\mathrm{HD}$ & $16 q 11.2-16 q 24.3$ & 46454000-90338000 & 43884000 & $11 ; 14$ \\
\hline 6 & $\mathrm{HD}$ & $16 q 11.2-16 q 24.3$ & $46454000-90338000$ & 43884000 & $11 ; 14$ \\
\hline 8 & $\mathrm{HD}$ & $16 q 11.2-16 q 24.3$ & 46454000-90338000 & 43884000 & $11 ; 14$ \\
\hline 9 & $\mathrm{HD}$ & $16 q 21.1-16 q 24.3$ & $50093000-89129000$ & 39036000 & $11 ; 14$ \\
\hline 15 & $\mathrm{HD}$ & $16 q 11.2-16 q 24.3$ & 46454000-90338000 & 43884000 & $11 ; 14$ \\
\hline 24 & $\mathrm{BD}$ & $16 q 12.1-16 q 12.1$ & $50232040-50913020$ & 680980 & $14 ; 16$ \\
\hline 25 & $\mathrm{HD}$ & $16 q 11.2-16 q 24.3$ & $46454000-90338000$ & 43884000 & $14 ; 16$ \\
\hline 28 & $\mathrm{HD}$ & $16 q 11.2-16 q 24.3$ & 46454000-90338000 & 43884000 & $14 ; 20$ \\
\hline 29 & $\mathrm{HD}$ & $16 q 11.2-16 q 24.3$ & $46454000-90338000$ & 43884000 & $14 ; 20$ \\
\hline 30 & $\mathrm{HD}$ & $16 q 11.2-16 q 24.3$ & $46454000-90338000$ & 43884000 & $14 ; 20$ \\
\hline 33 & $\mathrm{BD}$ & $16 q 12.1-16 q 12.1$ & $50777028-50812200$ & 35172 & $6 ; 14$ \\
\hline 35 & $\mathrm{HD}$ & $16 q 11.2-16 q 24.3$ & $46454000-90338000$ & 43884000 & Hyper + IGH sep \\
\hline 37 & $\mathrm{HD}$ & $16 q 11.2-16 q 24.3$ & $46454000-90338000$ & 43884000 & Hyper + IGH sep \\
\hline 40 & $\mathrm{BD}$ & $16 q 12.1-16 q 12.2$ & $50376741-52630833$ & 2254092 & Hyper + IGH sep \\
\hline 41 & $\mathrm{HD}$ & $16 q 11.2-16 q 24.3$ & 46454000-90338000 & 43884000 & Hyper + IGH sep \\
\hline 42 & $\mathrm{HD}$ & $16 q 12.1-16 q 12.1$ & $50193000-50827000$ & 634000 & Hyper + IGH sep \\
\hline 44 & $\mathrm{HD}$ & $16 q 11.2-16 q 24.3$ & 46454000-90338000 & 43884000 & Hyper \\
\hline 48 & $\mathrm{HD}$ & $16 q 12.1-16 q 12.2$ & $50123000-55838000$ & 5715000 & Hyper \\
\hline 49 & $\mathrm{BD}$ & $16 q 12.1-1612.1$ & $50290162-51082053$ & 791891 & Hyper \\
\hline 50 & $\mathrm{HD}$ & $16 q 11.2-16 q 24.3$ & 46454000-90338000 & 43884000 & Hyper \\
\hline 52 & $\mathrm{HD}$ & $16 q 11.2-16 q 24.3$ & $46454000-90338000$ & 43884000 & Hyper \\
\hline 54 & $\mathrm{HD}$ & $16 q 11.2-16 q 24.3$ & 46454000-90338000 & 43884000 & Hyper \\
\hline 61 & $\mathrm{HD}$ & $16 q 11.2-16 q 24.3$ & 46454000-90338000 & 43884000 & Hyper \\
\hline 63 & $\mathrm{HD}$ & $16 q 11.2-16 q 24.3$ & 46454000-90338000 & 43884000 & Hyper \\
\hline 66 & $\mathrm{HD}$ & $16 q 11.2-16 q 24.3$ & 46454000-90338000 & 43884000 & Tetraploid \\
\hline 67 & $\mathrm{HD}$ & $16 p 13.3-16 q 24.3$ & 0-90338345 & 90338345 & Monosomy 13/14 \\
\hline Case & $\mathrm{BIRC2}$ and $\mathrm{BIRC} 3$ & Location & Breakpoints & Size (bp) & Primary \\
\hline 6 & $\mathrm{HD}$ & $11 q 14.1-11 q 22.3$ & $79621665-108999346$ & 29377681 & $11 ; 14$ \\
\hline 21 & $\mathrm{BD}$ & $11 q 22.1-11 q 22.2$ & $101044665-102389301$ & 1344636 & $4 ; 14$ \\
\hline Case & TENT5C/FAM46C & Location & Breakpoints & Size (bp) & Primary \\
\hline 5 & $\mathrm{HD}$ & $1 \mathrm{p} 22.3-1 \mathrm{p} 12$ & 87833886-119707445 & 31873559 & $11 ; 14$ \\
\hline 6 & $\mathrm{HD}$ & 1p36.33-1p12 & 1-119990000 & 119989999 & $11 ; 14$ \\
\hline 9 & $\mathrm{HD}$ & 1p35.3-1p12 & 29234000-119991000 & 90757000 & $11 ; 14$ \\
\hline 15 & $\mathrm{HD}$ & 1p32.3-1p12 & 51107000-119761000 & 68654000 & $11 ; 14$ \\
\hline 21 & $\mathrm{HD}$ & 1p31.1-1p12 & 77992000-119733000 & 41741000 & $4 ; 14$ \\
\hline 23 & $\mathrm{HD}$ & $1 p 34.2-1 p 12$ & 42342000-121700000 & 79358000 & $4 ; 14$ \\
\hline 24 & $\mathrm{HD}$ & 1p13.3-1p12 & 110882000-120028000 & 9146000 & $14 ; 16$ \\
\hline 28 & $\mathrm{HD}$ & 1p32.1-1p12 & 58536000-119985000 & 61449000 & $14 ; 20$ \\
\hline 29 & $\mathrm{HD}$ & 1p31.1-1p12 & 75850000-118934000 & 43084000 & $14 ; 20$ \\
\hline 30 & Translocation to MYC & $1 \mathrm{p} 12$ & 117855602 & $\mathrm{~N} / \mathrm{A}$ & $14 ; 20$ \\
\hline 32 & $\mathrm{HD}$ & 1p31.1-1p12 & 70504000-119991000 & 49487000 & $6 ; 14$ \\
\hline 33 & $\mathrm{HD}$ & 1p36.33-1p12 & $1-119982000$ & 119981999 & $6 ; 14$ \\
\hline 34 & Translocation to MYC and MAFB & $1 \mathrm{p} 12$ & $117611301 ; 117746520$ & N/A & Hyper + IGH sep \\
\hline
\end{tabular}


Table 5 continued

\begin{tabular}{|c|c|c|c|c|c|}
\hline Case & TENT5C/FAM46C & Location & Breakpoints & Size (bp) & Primary \\
\hline 35 & $\mathrm{HD}$ & $1 p 33-1 p 12$ & 49064000-119989000 & 70925000 & Hyper + IGH sep \\
\hline 37 & $H D$ & $1 \mathrm{p} 22.2-1 \mathrm{p} 12$ & 89819000-118483000 & 28664000 & Hyper + IGH sep \\
\hline 40 & $\mathrm{HD}$ & 1p13.1-1p12 & 116616000-119990000 & 3374000 & Hyper + IGH sep \\
\hline 53 & $\mathrm{HD}$ & 1p31.1-1p12 & 77694018-119983000 & 42288982 & Hyper \\
\hline 56 & Translocation to MYC & $1 \mathrm{p} 12$ & $117615759 ; 117851083$ & N/A & Hyper \\
\hline 58 & Translocation to IL16 & $1 \mathrm{p} 12$ & $117592488 ; 117745524$ & N/A & Hyper \\
\hline 59 & Translocation to MYC & $1 \mathrm{p} 12$ & 117670599 & N/A & Hyper \\
\hline 60 & $\mathrm{HD}$ & 1p22.1-1p12 & 92935000-119981000 & 27046000 & Hyper \\
\hline 61 & Translocation to MYC & $1 \mathrm{p} 12$ & $117653222 ; 117665080$ & N/A & Hyper \\
\hline 64 & $\mathrm{HD}$ & 1p31.3-1p12 & 67860000-118597000 & 50737000 & Hyper \\
\hline 66 & $\mathrm{HD}$ & 1p36.33-1p12 & 1-119990000 & 119989999 & Tetraploid \\
\hline 67 & $\mathrm{HD}$ & 1p36.33-1p12 & 1-119991000 & 119990999 & Monosomy 13/14 \\
\hline Case & $\mathrm{CDKN2C}$ and FAF1 & Location & Breakpoints & Size (bp) & Primary \\
\hline 5 & BD & $1 p 32.3-1$ p32.3 & $50884258-51012825$ & 128567 & $11 ; 14$ \\
\hline 6 & $\mathrm{HD}$ & 1p36.33-1p12 & 1-119990000 & 119989999 & $11 ; 14$ \\
\hline 9 & $\mathrm{HD}$ & 1p35.3-1p12 & 29234000-119991000 & 90757000 & $11 ; 14$ \\
\hline 10 & $\mathrm{HD}$ & 1p32.3-1p32.3 & 50402893-50989867 & 586974 & $11 ; 14$ \\
\hline 15 & BD & 1 p32.3-1p32.3 & 50599579-51106763 & 507184 & $11 ; 14$ \\
\hline 21 & $\mathrm{HD}$ & 1p32.2-1p31.1 & $50276000-73879000$ & 23603000 & $4 ; 14$ \\
\hline 22 & $\mathrm{BD}$ & 1p32.3-1p32.3 & $50924750-50971658$ & 46908 & $4 ; 14$ \\
\hline 23 & $\mathrm{HD}$ & 1p34.2-1p12 & $42342000-121700000$ & 79358000 & $4 ; 14$ \\
\hline 24 & HD FAF1 only & 1p33-1p32.3 & $49951000-50606000$ & 655000 & $14 ; 16$ \\
\hline 33 & $\mathrm{HD}$ & 1p36.33-1p12 & 1-119982000 & 119981999 & $6 ; 14$ \\
\hline 34 & $\mathrm{HD}$ & 1p32.3-1p12 & 50750000-117611000 & 66861000 & Hyper + IGH sep \\
\hline 35 & $\mathrm{HD}$ & $1 p 33-1 p 12$ & 49064000-119989000 & 70925000 & Hyper + IGH sep \\
\hline 40 & $\mathrm{HD}$ & 1p33-1p13.3 & 49723000-109237000 & 59514000 & Hyper + IGH sep \\
\hline 51 & $H D$ & 1p33-1p31.3 & $50018770-65125485$ & 15106715 & Hyper \\
\hline 52 & BD & 1 p32.3-1p32.3 & $50925212-51007221$ & 82009 & Hyper \\
\hline 58 & $H D$ & 1p32.3-1p13.3 & $50467681-107513627$ & 57045946 & Hyper \\
\hline 60 & $\mathrm{HD}$ & 1p34.1-1p32.2 & $45515071-55049538$ & 9534467 & Hyper \\
\hline 66 & $\mathrm{HD}$ & 1p36.33-1p12 & 1-119990000 & 119989999 & Tetraploid \\
\hline 67 & $\mathrm{HD}$ & 1p36.33-1p12 & 1-119991000 & 119990999 & Monosomy 13/14 \\
\hline Case & MAP3K14 & Location & Breakpoints & Size (bp) & Primary \\
\hline 2 & $\mathrm{HD}$ & $17 q 21.31-17 q 21.32$ & $44943000-47142000$ & 2199000 & $11 ; 14$ \\
\hline 36 & $H D$ & $17 q 21.31-17 q 21.31$ & $44583000-45982000$ & 1399000 & Hyper + IGH sep \\
\hline 44 & $\mathrm{HD}$ & 17p13.3-17q21.31 & $1-45734287$ & 45734286 & Hyper \\
\hline 45 & Gain & $17 q 21.31-17 q 21.31$ & $45191000-45926000$ & 735000 & Hyper \\
\hline
\end{tabular}




\begin{tabular}{|c|c|c|c|c|c|}
\hline Case & NFKB1 or NFKB2 & Location & Breakpoints & Size (bp) & Primary \\
\hline 6 & HD NFKB1 & $4 q 13.2-4 q 26$ & $65930271-115942883$ & 50012612 & $11 ; 14$ \\
\hline 23 & HD NFKB1 & $4 p 14-4 q 35.2$ & 36402000-189875000 & 153473000 & $4 ; 14$ \\
\hline 33 & HD NFKB1 & $4 p 16.3-4 q 35.2$ & $1-190214555$ & 190214554 & $6 ; 14$ \\
\hline 34 & HD NFKB2 & 10q24.1-10q26.3 & 97005000-133797422 & 36792422 & Hyper + IGH sep \\
\hline 40 & HD NFKB1 & $4 p 16.3-4 q 35.2$ & 1-190214555 & 190214554 & Hyper + IGH sep \\
\hline 44 & HD NFKB1 & $4 q 13.3-4 q 31.3$ & $73221000-150520000$ & 77299000 & Hyper \\
\hline 46 & HD NFKB2 & 10q24.32-10q24.33 & 101899000-103362000 & 1463000 & Hyper \\
\hline 47 & HD NFKB2 & 10q24.32-10q24.32 & $102148932-102721927$ & 572995 & Hyper \\
\hline 51 & HD NFKB1 & $4 p 16.3-4 q 35.2$ & $1-190214555$ & 190214554 & Hyper \\
\hline 65 & HD NFKB2 & 10q24.32-10q25.1 & $102399071-104266176$ & 1867105 & Hyper \\
\hline 66 & HD NFKB1 & $4 p 16.3-4 q 35.2$ & 1-190214555 & 190214554 & Tetraploid \\
\hline 66 & HD NFKB2 & 10p15.3-10q26.3 & $1-133797422$ & 133797421 & Tetraploid \\
\hline 67 & HD NFKB2 & $10 q 11.21-10 q 26.3$ & 42354000-133797422 & 91443422 & Monosomy $13 / 14$ \\
\hline Case & TRAF2 or TRAF3 & Location & Breakpoints & Size (bp) & Primary \\
\hline 8 & HD TRAF3 & $14 q 22.3-14 q 32.33$ & 56254000-104990000 & 48736000 & $11 ; 14$ \\
\hline 23 & HD TRAF3 & $14 q 11.2-14 q 32.33$ & 19958000-105864169 & 85906169 & $4 ; 14$ \\
\hline 32 & BD TRAF3 & $14 q 32.32-14 q 32.32$ & $102754161-102809688$ & 55527 & $6 ; 14$ \\
\hline 37 & HD TRAF3 & $14 q 24.3-14 q 32.33$ & $77344000-105590563$ & 28246563 & Hyper + IGH sep \\
\hline 44 & HD TRAF3 & $14 q 21.1-14 q 32.33$ & 39707000-107043718 & 67336718 & Hyper \\
\hline 47 & HD TRAF3 & $14 q 32.32-14 q 32.32$ & $102845410-102902550$ & 57140 & Hyper \\
\hline 52 & BD TRAF3 & $14 q 32.32-14 q 32.32$ & $102722216-102790013$ & 67797 & Hyper \\
\hline 60 & BD TRAF3 & $14 q 32.32-14 q 32.32$ & 102741220-102888391 & 147171 & Hyper \\
\hline 62 & BD TRAF3 & $14 q 32.31-14 q 32.32$ & $102680377-102913558$ & 233181 & Hyper \\
\hline 67 & BD TRAF3 & $14 q 32.32-14 q 32.32$ & $102841855-102878463$ & 36608 & Monosomy 13/14 \\
\hline 67 & HD TRAF2 & $9 q 34.3-9 q 34.3$ & 136828276-136921241 & 92965 & Monosomy $13 / 14$ \\
\hline Case \# & CRBN or IKZF1 or IKZF3 & Location & Breakpoints & Size (bp) & Primary \\
\hline 8 & HD IKZF3 & $17 q 12-17 q 21.31$ & $35371000-44480000$ & 9109000 & $11 ; 14$ \\
\hline 17 & HD CRBN & $3 p 26.3-3 p 26.2$ & 2738159-3194829 & 456670 & $4 ; 14$ \\
\hline 22 & HD CRBN & $3 p 26.3-3 q 22.1$ & $1-130531000$ & 130530999 & $4 ; 14$ \\
\hline 28 & HD CRBN & $3 p 26.3-3 p 25.2$ & $1-12659000$ & 12658999 & $14 ; 20$ \\
\hline 41 & HD CRBN & $3 p 26.3-3 p 24.1$ & $1-28213000$ & 28212999 & Hyper + IGH sep \\
\hline 44 & HD IKZF3 & 17p13.3-17q21.31 & $1-45734287$ & 45734286 & Hyper \\
\hline 58 & HD CRBN & $3 p 26.3-3 p 26.1$ & $1-7110000$ & 7109999 & Hyper \\
\hline 58 & IKZF1 Gain + insertion to 10q25.2 & $17 p 12.2$ & $50207542-50430511$ & 222969 & Hyper \\
\hline
\end{tabular}

Abnormalities of genes of known clinical significance in MM

Large gains of chromosome material are not indicated

$H D$ heterozygous deletion, $B D$ biallelic deletion indicated in bold, cytogenetic band and location in GRCh38 
sequences promoting aberrant $M Y C$ gene expression, which may be targeted by BRD4 inhibitors in $\mathrm{MM}^{10}$. Identification of $M Y C$ rearrangements using a break-apart probe strategy resulted in a $50.0 \%$ false negative rate in our patient cohort. Whether these false negative insertion cases have the same prognostic implication as other $M Y C$ rearrangements remains unknown.

Two cases had deletions of the TP53 gene region that were not identified by FISH. For cases 4 and 21, MPseq identified a deletion of TP53 $(5.6 \mathrm{Mb}$ in case $4,2.7 \mathrm{Mb}$ in case 21). Interestingly, for case 21 , MPseq also identified a translocation involving TP53 (to 4q32.1). Both cases were scored as having two copies of TP53 by FISH and represent false negative results due the location of the deletion in relation to the FISH footprint in case 4 and the TP53 translocation in combination with the deletion in case 21. Although these cases had missed high risk abnormalities, the mSMART risk did not change since those cases also had additional high risk abnormalities [1q gain for cases 4 and $t(4 ; 14)$ for case 21$]$. For case 4 , a separate NGS assay analyzing SNVs identified a pathogenic TP53 mutation [Chr17(GRCh37):g.7577111 G > T; NM_001126113.2 (TP53):c.827 C > A; p.Ala276Asp] located in the DNAbinding domain and in vitro functional data predicts that this variant results in non-functional p53 protein $^{44}$. TP53 has been found to be mutated in $3-16 \%$ of $\mathrm{NDMM}^{6,45-47}$ with a higher frequency in RRMM ${ }^{48-50}$. TP53 mutations in combination with $17 \mathrm{p}$ deletions are associated with double hit MM with reduced overall, progression-free and relapse-free survival ${ }^{51}$. Therefore, this missed TP53 deletion fails to identify the presence of a likely double hit $\mathrm{MM}$ in patient 4. The combination of a rearrangement and deletion also likely represents a double hit MM abnormality in case 21 .

The CCND1/IGH dual color, dual fusion probe set is used to identify $C C N D 1 / I G H$ rearrangements. However, three copies of CCND1 in the absence of IGH fusion can indicate trisomy 11 or non-IGH CCND1 rearrangements. MPseq identified three CCND1 rearrangements including case 4 (IGL/CCND1), case 43 (IGK/CCND1) described more fully in Peterson, et al..$^{52}$ and case 57 (BRINP3/ $C C N D 1)$. FISH also identified amplification of CCND1 in case 4, three copies of CCND1 in case 43 and a normal signal pattern for $C C N D 1$ in case 57 . The CCND1 rearrangement identified in case 57 was a complex translocation between 1q31.1 and 11q13.3 consisting of four junctions and deletions of $\sim 100 \mathrm{~kb}$ at both ends. Through this complex event, CCND1 is brought into close proximity to the $3^{\prime}$ end of BRINP3, while the balancing set of junctions brings the $5^{\prime}$ end of BRINP3 near 11q24.3. Additionally, the derivative chromosome containing CCND1 has been copied. Overall this would result in three copies of CCND1, two of which have been translocated near the $3^{\prime}$ end of BRINP3. This case demonstrates how MPseq is able to determine complex rearrangements involving important genes without prior knowledge of the junction partner location.

Although immunoglobulin lambda rearrangements have been recently reported in association with poor prognosis ${ }^{3}$, light chain rearrangements are typically not evaluated in the diagnostic work up of $M M$ in most clinical genomics laboratories. Using MPseq data, we identify 10 cases (14.3\% of entire cohort) with IGL rearrangements with five of these cases with standard risk cytogenetic results. IGL rearrangements and other focal deletions of clinical significance are typically not evaluated by FISH. Given the high rate of false-negative $M Y C$ rearrangements and inability to appreciate all abnormalities of clinical significance, we demonstrate that MPseq has increased clinical value compared to FISH in characterizing genomic abnormalities in PCNs.

\section{Acknowledgements}

Research reported in this publication was supported with contributions from the Mayo Clinic Department of Laboratory Medicine and Pathology with support from the Marion Schwartz Career Development Award in Multiple Myeloma, Center for Individualized Medicine and the National Cancer Institute of the National Institutes of Health under Award Number P50CA186781 from the Mayo Clinic Multiple Myeloma Specialized Program of Research Excellence. The content is solely the responsibility of the authors and does not necessarily represent the official views of the National Institutes of Health.

\section{Author details}

${ }^{1}$ Center for Individualized Medicine-Biomarker Discovery, Mayo Clinic, Rochester, MN, USA. ${ }^{2}$ Division of Laboratory Genetics, Department of Laboratory Medicine and Pathology, Mayo Clinic, Rochester, MN, USA. ${ }^{3}$ Division of Hematopathology, Department of Laboratory Medicine and Pathology, Mayo Clinic, Rochester, MN, USA. ${ }^{4}$ Division of Hematology, Department of Internal Medicine, Mayo Clinic, Scottsdale, AZ, USA. ${ }^{5}$ Division of Hematology, Department of Internal Medicine, Mayo Clinic, Rochester, MN, USA

\section{Conflict of interest}

S.K.: AbbVie, Celgene, Janssen, Merck, Novartis, Roche, Amgen, Sanofi, and Takeda (research funding, consulting) and Adaptive (honoraria). K.S.: consultant for Bristol-Myers Squibb, Celgene, Amgen, Janssen, Takeda, and Roche.

Algorithms described in this paper are licensed to WholeGenome LLC, owned by G.V.

\section{Publisher's note}

Springer Nature remains neutral with regard to jurisdictional claims in published maps and institutional affiliations.

Supplementary Information accompanies this paper at (https://doi.org/ 10.1038/s41408-019-0255-z).

Received: 17 July 2019 Revised: 21 October 2019 Accepted: 4 November 2019

Published online: 16 December 2019

\footnotetext{
References

1. Howlader, N. et al. Improved estimates of cancer-specific survival rates from population-based data. J. Natl Cancer Inst.102, 1584-1598 (2010).
} 
2. Rajkumar, S. V. Multiple myeloma: 2018 update on diagnosis, risk-stratification, and management. Am. J. Hematol. 93, 981-1114 (2018).

3. Barwick, B. G. et al. Multiple myeloma immunoglobulin lambda translocations portend poor prognosis. Nat. Commun. 10, 1911 (2019).

4. Chapman, M. A. et al. Initial genome sequencing and analysis of multiple myeloma. Nature. 471, 467-472 (2011).

5. Egan, J. B. et al. Whole-genome sequencing of multiple myeloma from diagnosis to plasma cell leukemia reveals genomic initiating events, evolution, and clonal tides. Blood. 120, 1060-1066 (2012).

6. Lohr, J. G. et al. Widespread genetic heterogeneity in multiple myeloma: implications for targeted therapy. Cancer Cell. 25, 91-101 (2014).

7. Miller, A. et al. High somatic mutation and neoantigen burden are correlated with decreased progression-free survival in multiple myeloma. Blood Cancer J. 7, e612 (2017).

8. Walker, B. A. et al. Identification of novel mutational drivers reveals oncogene dependencies in multiple myeloma. Blood. 132, 587-97. (2018).

9. Walker, B. A. et al. APOBEC family mutational signatures are associated with poor prognosis translocations in multiple myeloma. Nat. Commun. 6, 6997 (2015).

10. Affer, M. et al. Promiscuous MYC locus rearrangements hijack enhancers but mostly super-enhancers to dysregulate MYC expression in multiple myeloma. Leukemia. 28, 1725-35. (2014).

11. Misund K. K. et al. MYC dysregulation in the progression of multiple myeloma. Leukemia (2019). https://www.ncbi.nlm.nih.gov/pubmed/31439946. [Epub ahead of print]

12. Manier, S. et al. Genomic complexity of multiple myeloma and its clinical implications. Nat. Rev. Clin. Oncol. 14, 100-13. (2017).

13. Bolli, N. et al. Analysis of the genomic landscape of multiple myeloma highlights novel prognostic markers and disease subgroups. Leukemia. 32, 2604-16. (2018).

14. Rajkumar S. V. mSMART stratification for myeloma and risk-adapted therapy. www.msmart.org

15. Pugh, T. J. et al. Assessing genome-wide copy number aberrations and copyneutral loss-of-heterozygosity as best practice: an evidence-based review from the Cancer Genomics Consortium working group for plasma cell disorders. Cancer Genet-Ny 228, 184-96. (2018).

16. Kumar, S. K. \& Rajkumar, S. V. The multiple myelomas - current concepts in cytogenetic classification and therapy. Nat. Rev. Clin. Oncol. 15, 409-21. (2018).

17. Sonneveld, P. et al. Treatment of multiple myeloma with high-risk cytogenetics: a consensus of the International Myeloma Working Group. Blood. 127, 2955-2962 (2016).

18. Aypar, U. et al. Mate pair sequencing improves detection of genomic abnormalities in acute myeloid leukemia. Eur. J. Haematol. 102, 87-96 (2019).

19. King R. L., et al. False-negative rates for MYC FISH probes in B-cell neoplasms. Haematologica. 104, e248-e251 (2019). https://doi.org/10.3324/ haematol.2018.207290, https:/www.ncbi.nlm.nih.gov/pubmed/?term=Falsenegative+rates+for+MYC+FISH+probes+in+B-cell+neoplasms. [Epub 6 Dec 2018].

20. Peterson, J. F. et al. Characterization of a cryptic IGH/CCND1 rearrangement in a case of mantle cell lymphoma with negative CCND1 FISH studies. Blood Adv. 3, 1298-302. (2019)

21. Peterson J. F., et al. Use of mate-pair sequencing to characterize a complex cryptic BCR/ABL1 rearrangement observed in a newly diagnosed case of chronic myeloid leukemia. Hum. Pathol. 89, 109-114 (2019). https:/doi.org/ 10.1016/j.humpath.2018.09.010, https://www.ncbi.n/m.nih.gov/pubmed/? term $=$ Use + of + matepair + sequencing + to + characterize $+\mathrm{a}+$ complex +cryptic+BCR\%2FABL1+rearrangement+observed +in +a+newly +diagnosed+case+of+chronic+myeloid+leukemia. [Epub 26 Sep 2018].

22. Peterson J. F., et al. Detection of a cryptic NUP214/ABL1 gene fusion by matepair sequencing (MPseq) in a newly diagnosed case of pediatric Tlymphoblastic leukemia. Cold Spring Harb. Mol. Case Stud. 5, pii: a003533. (2019). https://doi.org/10.1101/mcs.a003533, https://www.ncbi.nlm.nih.gov/ pubmed/?term=Detection+of+a+cryptic+NUP214\%2FABL1+gene+fusion +by+mate-pair+sequencing +(MPseq) +in+a+newly+diagnosed+case+of +pediatric+T-lymphoblastic+leukemia.

23. Walker, B. A. et al. Translocations at $8 \mathrm{q} 24$ juxtapose MYC with genes that harbor superenhancers resulting in overexpression and poor prognosis in myeloma patients. Blood. Cancer J. 4, e191 (2014).

24. Walker, B. A. et al. Characterization of IGH locus breakpoints in multiple myeloma indicates a subset of translocations appear to occur in pregerminal center B cells. Blood. 121, 3413-3419 (2013).
25. Baughn, L. B. et al. Differences in genomic abnormalities among African individuals with monoclonal gammopathies using calculated ancestry. Blood Cancer J. 8, 96 (2018).

26. Jang, J. S. et al. Molecular signatures of multiple myeloma progression through single cell RNA-Seq. Blood Cancer J. 9, 2 (2019).

27. Johnson, S. H. et al. SVAtools for junction detection of genome-wide chromosomal rearrangements by mate-pair sequencing (MPseq). Cancer Genet 221, 1-18 (2018).

28. Smadbeck J. B., et al. Copy number variant analysis using genome-wide matepair sequencing. Genes Chromosomes Cancer. 57, 459-470 (2018). https://doi. org/10.1002/gcc.5, https://www.ncbi.nlm.nih.gov/pubmed/29726617. [Epub 30 Jul 2019].

29. Drucker, T. M. et al. BIMA V3: an aligner customized for mate pair library sequencing. Bioinformatics. 30, 1627-1629 (2014).

30. Gaitatzes, A.t Johnson, S. H., Smadbeck, J. B. \& Vasmatzis, G. Genome U-Plot: a whole genome visualization. Bioinformatics. 34, 1629-34. (2018).

31. Lee, N. et al. Discrepancies between the percentage of plasma cells in bone marrow aspiration and BM biopsy: Impact on the revised IMWG diagnostic criteria of multiple myeloma. Blood Cancer J. 7, e530 (2017).

32. Demchenko, Y. et al. Frequent occurrence of large duplications at reciprocal genomic rearrangement breakpoints in multiple myeloma and other tumors. Nucleic Acids Res 44, 8189-8198 (2016).

33. Keats, J. J. et al. Promiscuous mutations activate the noncanonical NF-kappaB pathway in multiple myeloma. Cancer Cell. 12, 131-144 (2007).

34. Zhu, Y. X. et al. Loss of FAM46C promotes cell survival in myeloma. Cancer Res. 77, 4317-27. (2017).

35. Jimenez, $\mathrm{C}$. et al. A next-generation sequencing strategy for evaluating the most common genetic abnormalities in multiple myeloma. J. Mol. Diagn. 19, 99-106 (2017).

36. Bolli, N. et al. A DNA target-enrichment approach to detect mutations, copy number changes and immunoglobulin translocations in multiple myeloma. Blood Cancer J. 6, e467 (2016).

37. White, B. S. et al. A multiple myeloma-specific capture sequencing platform discovers novel translocations and frequent, risk-associated point mutations in IGLL5. Blood Cancer J. 8, 35 (2018).

38. Goldsmith S. R., et al. Next generation sequencing-based validation of the revised international staging system for multiple myeloma: an analysis of the MMRF CoMMpass Study. Clin. Lymphoma Myeloma Leuk. 19, 285-289 (2019). https://doi.org/10.1016/j.clml.2019.01.003, https://www.ncbi.nlm.nih.gov/ pubmed/30792096. [Epub 19 Jan 2019].

39. Davila-Rodriguez, M. I. et al. Constitutive heterochromatin polymorphisms in human chromosomes identified by whole comparative genomic hybridization. Eur. J. Histochem. 55, e28 (2011).

40. Sidana, S. et al. Rapid assessment of hyperdiploidy in plasma cell disorders using a novel multi-parametric flow cytometry method. Am. J. Hematol. 94 424-30. (2019).

41. Sidana, S. et al. Tetraploidy is associated with poor prognosis at diagnosis in multiple myeloma. Am. J. Hematol. 94, E117-E20. (2019).

42. Shou, Y. et al. Diverse karyotypic abnormalities of the c-myc locus associated with c-myc dysregulation and tumor progression in multiple myeloma. Proc. Natl Acad. Sci. USA 97, 228-233 (2000).

43. Glitza, I. C. et al. Chromosome 8q24.1/c-MYC abnormality: a marker for highrisk myeloma. Leuk. Lymphoma. 56, 602-607 (2015).

44. Kato, S. et al. Understanding the function-structure and function-mutation relationships of p53 tumor suppressor protein by high-resolution missense mutation analysis. Proc. Natl Acad. Sci USA 100, 8424-8429 (2003).

45. Bolli, N. et al. Heterogeneity of genomic evolution and mutational profiles in multiple myeloma. Nat. Commun. 5, 2997 (2014).

46. Kortum, K. M. et al. Longitudinal analysis of 25 sequential sample-pairs using a custom multiple myeloma mutation sequencing panel (M(3)P). Ann. Hematol. 94, 1205-1211 (2015).

47. Walker, B. A. et al. Mutational spectrum, copy number changes, and outcome: results of a sequencing study of patients with newly diagnosed myeloma. J. Clin. Oncol. 33, 3911-3920 (2015).

48. Weinhold, N. et al. Clonal selection and double-hit events involving tumor suppressor genes underlie relapse in myeloma. Blood. 128, 1735-1744 (2016).

49. Xiong, W. et al. An analysis of the clinical and biologic significance of TP53 loss and the identification of potential novel transcriptional targets of TP53 in multiple myeloma. Blood. 112, 4235-4246 (2008). 
50. Chng, W. J. et al. Clinical significance of TP53 mutation in myeloma. Leukemia. 21, 582-584 (2007).

51. Walker, B. A. et al. A high-risk, Double-Hit, group of newly diagnosed myeloma identified by genomic analysis. Leukemia. 33, 159-70. (2019).
52. Peterson, J. F. et al. Whole genome mate-pair sequencing of plasma cell neoplasm as a novel diagnostic strategy: a case of unrecognized t (2; 11) structural variation. Clin. Lymphoma, Myeloma Leuk. 19, 598-602 (2019) 